\title{
A Multiuser Receiver for Code Division Multiple Access Communications over Multipath Channels
}

\author{
Urs Fawer, Member, IEEE, and Behnaam Aazhang, Senior Member, IEEE
}

\begin{abstract}
A multiuser communication system is considered where $K$ users share a channel with multipath propagation by using code division for multiple access. Data modulation is carried out by binary phase shift keying and direct sequence spread spectrum signaling. The microcellular communication media is modeled as a frequency selective fading channel with multipath propagation. The multipath diversity of the received signals from the $K$ users is exploited by a bank of $K$ Rake correlators. Algorithms based on the maximum likelihood rule have been developed for estimating the complex channel coefficients as well as for detection of the desired data packets from the sufficient statistics provided by the Rake correlators. The performance of the resulting multiuser detector is evaluated analytically and via Monte Carlo simulations. The results indicate that the estimator of the channel coefficients has a variance close to the Cramér-Rao lower bound, and that the proposed multiuser detector is capable of eliminating the near-far effect as well as processing the signals propagated through multiple paths.
\end{abstract}

Keywords-Multiuser detection, code division multiple access (CDMA), Rake, Gauss-Seidel iteration, Expectation Maximization (EM).

\section{INTRODUCTION}

Code division multiple access (CDMA) communication has been a popular random access technique in the research community for many years. There are now several operational CDMA systems for both military and commercial applications including satellite networking, cellular mobile radio systems, and indoor wireless communications [1-3].

Among the many research problems posed in the area of code division multiple access communications, the near-far problem is of particular interest since it has always been considered a limiting factor in the application of this multiple access scheme. The near-far effect exists when a weak received signal is interfered with by one or more strong signals such that the effective cross-correlations among the signals degrade the performance of the conventional CDMA receiver. One solution to combat this problem is multiuser detection where the receiver exploits the knowledge of the spread spectrum signals of the interfering users to eliminate

Paper approved by Gordon L. Stüber, the Editor for Spread Spectrum of the IEEE Communications Society. Manuscript received: November 2, 1993; revised: June 29, 1994. This work was supported in part by the Swiss PTT General Directorate, $R \& D$, Mobile Communications (VD 2), and by the Advanced Technology Program of the Texas Higher Education Coordinating Board under Grant 003604018. This paper was presented in part at the Communication Theory Mini-Conference, GLOBECOM'92, Orlando, Florida, December 1992.

U. Fawer was with the Communication Technology Laboratory of the Swiss Federal Institute of Technology in Zürich, Switzerland. He is now with Faselec AG (Philips Semiconductors), Binzstrasse 44, CH-8045 Zürich, Switzerland.

B. Aazhang is with the Department of Electrical and Computer Engineering, Rice University, Houston, Texas 77251-1892.

IEEE Log Number 9411622. the near-far effect. 'The interest in the problem of multiuser detection was motivated by the work of Verdú [4] where he devised both the minimum error probability detector and the maximum likelihood sequence detector for CDMA systems over Gaussian noise channels. Many suboptimum algorithms, which are computationally less intensive than the optimum ones, followed the development of the optimum detectors [5-10]. There are still several open problems in the area of multiuser detection when one considers CDMA systems which do not operate over the standard additive white Gaussian noise channel [11-15].

In this paper, we consider multiple access communications over multipath fading channels. There are many radio channels that exhibit severe multipath propagation which causes frequency selective fading $[16,17]$. In the time domain, such a channel property corresponds to delay or multipath diversity that can be exploited at the receiver by properly processing the incoming signals. The detection problem becomes even more challenging when more than one user share the multipath fading channel. Examples of such scenarios include cellular mobile radio systems, indoor wireless communications and wireless local area networks.

This paper addresses the problem of multiuser detection in multipath fading channels. In particular, we consider a CDMA system where $K$ users share a channel with $L$ propagation paths per user. The relative propagation times of the $L$ paths are assumed to be known (or estimated). It is shown that the outputs of a bank of $K \times L$ matched filters or, equivalently, the outputs of $K$ Rake correlators with $L$ arms, observed over the length of the data packets, form the sufficient statistics $[11,18,19]$. The joint maximization of the likelihood function with respect to channel parameters and data packets has a computational complexity too high to be of practical use. We devise an iterative scheme for the joint estimation and detection problem based on the Gauss-Seidel iteration [20,21]. In one step of the iteration, we apply either the maximum likelihood technique to formulate a least squares problem of size $K L$ or the Expectation Maximization (EM) algorithm (see [21] and references therein) to decompose the problem of estimating the set of $K L$ channel parameters into $K L$ decoupled parameter estimation problems. For the other step of the iteration, we make use of the multistage detection algorithm [7] to demodulate the data packets of the $K$ users.

The performance of the proposed multiuser receiver is examined by Monte Carlo simulations and analytical approximations of the bit-error probability. Our numerical results indicate that the estimation process of the channel coefficients performs with a variance close to the CramérRao lower bound. Furthermore, the receiver is shown to be (c) 1995 IEEE 


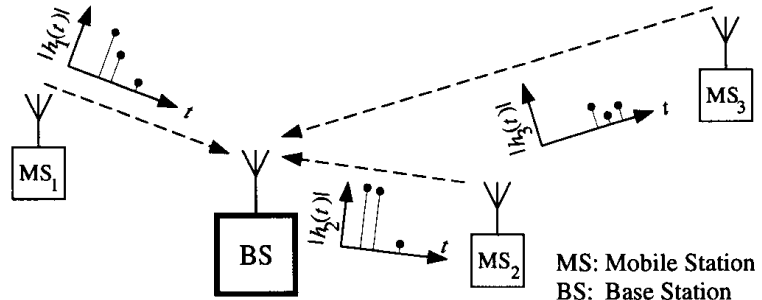

Fig. 1. Multiple access communication system with multipath propagation.

superior in performance (i.e., average bit-error probability) to the conventional receiver which is a bank of $K$ Rake receivers with no post-processing. The numerical results show that the multiuser detector exploits the multipath diversity and exhibits no near-far effects.

The following section contains the system description. Section III treats the derivation of the multiuser diversity receiver via a direct maximum likelihood approach. In Section IV, an iterative algorithm based on the GaussSeidel scheme is derived that applies the EM and the multistage detection algorithms. Section V describes a sequential and adaptive implementation of the proposed multiuser and multipath receiver that is suitable for slowly varying channels. The performance analysis in Section VI contains analytical expressions as well as numerical results. The conclusions are finally drawn in Section VII.

\section{System Description}

We consider a CDMA system where $K$ users transmit data over a multipath fading channel with possibly $K L$ distinct propagation paths as illustrated in Fig. 1. The impulse response of the channel that the signal of the $k^{\text {th }}$ user propagates through is written as

$$
h_{k}(t)=\sum_{l=1}^{L} h_{k, l} \delta\left(t-\tau_{k, l}\right)
$$

where $h_{k, l}$ is the complex channel fading coefficient and $\tau_{k, l}$ is the delay that the $k^{\text {th }}$ signal experiences passing through the $l^{t h}$ path. The $K$ data packets of length $2 P+1$ are transmitted using spread spectrum signaling with binary phase shift keying (BPSK) modulation. The spectrumspreading waveform of the $k^{t h}$ user can be written as

$$
a_{k}(t)=\sum_{n=0}^{N_{c}-1} a_{n}^{(k)} \Pi_{T_{c}}\left(t-n T_{c}\right),
$$

where $\Pi_{T_{c}}(t)$ is a unit rectangular pulse with a duration of one chip $T_{c}$ and $a_{n}^{(k)} \in\{-1,+1\}$ is a spreading code symbol. The multiplication of this waveform with the modulated data signal is referred to as direct sequence spread spectrum modulation. It is assumed that a spreading code of length $N_{c}$ is assigned to a single bit, that is, the bit period $T_{b}$ is equal to $N_{c} T_{c}$. The base-band form ${ }^{1}$ of the received signal at a particular receiver for this multiuser and multipath system is given by

$$
r(t)=s(t, \mathcal{B})+n(t),
$$

where

$$
s(t, \mathcal{B})=\sum_{i=-P}^{P} \sum_{k=1}^{K} \sum_{l=1}^{L} h_{k, l} \sqrt{\frac{E_{b}}{T_{b}}} b_{k}^{(i)} a_{k}\left(t-\tau_{k, l}-i T_{b}\right) .
$$

The base-band channel noise $n(t)$ is modeled by a zero mean, white complex Gaussian process with a flat, twosided power spectral density with height $\mathcal{N}_{0} / 2$ for both the real and imaginary parts. In this model, $\mathcal{B}=\left[b_{k}^{(i)}\right]_{K \times(2 P+1)}$ represents the matrix of data packets where $b_{k}^{(i)} \in\{-1,+1\}$ is the $i^{\text {th }}$ information bit of the $k^{t h}$ user and $E_{b}$ represents the bit energy of each of the $K$ users. Since the energies of the $K$ transmitted signals are assumed to be equal, the near-far problem is formulated by considering different path attenuation variables. It is convenient to define the attenuation variables

$\alpha_{k, l}=\sqrt{\frac{E_{b}}{T_{b}}} h_{k, l}$, for $k=1,2, \ldots, K$, and $l=1,2, \ldots, L$.

Throughout this work, it is assumed that the $\alpha_{k, l}$ 's do not vary for the duration of one data packet. This condition does hold in many applications since the observation interval, that is the packet length in the system described above, is often much shorter than the coherence time of the channel, which characterizes the time-variant behavior of the propagation media. However, the sequential scheme described in Section $\mathrm{V}$ is designed for time-variant channels.

Throughout the paper we assume that the relative delays of the $K$ transmitting signals and the relative propagation time of all the paths have been estimated, i.e., $\tau_{k, \text { l's for all }}$ $k$ and $l$ have been obtained through a synchronization device $[22,23]$. For convenience and without loss of generality, we chose the indices of the $K$ users and its related propagation paths in such a way that the inequalities $\tau_{k, l}<\tau_{k, l^{\prime}}$, $\forall l^{\prime}>l$, and $\tau_{k, 1} \leq \tau_{k^{\prime}, 1}$ when $k^{\prime}>k$ are satisfied. Moreover, to simplify the problem, we assume that the following conditions hold: $\tau_{K, 1}-\tau_{1,1}<T_{b}$ and $\tau_{k, L}-\tau_{k, 1}<T_{b} / 2$, $\forall k$

\section{Multiuser Multipath Diversity Detector}

We are interested in detecting the data packets $\mathcal{B}$ of the $K$ users while assuming that the complex attenuation variables are unknown. It is convenient to formulate a vector notation since the problem in hand deals with the estimation of $K L$ attenuation variables and detection of $K(2 P+1)$ data bits. The $K L$ dimensional column vector ${ }^{2}$

\footnotetext{
${ }^{1}$ The bandpass signal $x(t)$ centered at the carrier frequency $f_{c}$ can be expressed by means of its equivalent base-band signal (i.e., the complex envelope) $y(t)$, as follows: $x(t)=\sqrt{2} \Re\left\{y(t) \exp \left(j 2 \pi f_{c} t\right)\right\}$ where $\Re\{\cdot\}$ represents the real part of its argument.
}

2 The transpose operator is denoted by $(\cdot)^{\top}$ 
$\boldsymbol{\alpha}=\left(\alpha_{1,1}, \alpha_{1,2}, \ldots, \alpha_{1, L}, \alpha_{2,1}, \ldots, \alpha_{K, L}\right)^{\top}$ represents the set of attenuation variables. The matrix of data packets $\mathcal{B}$ is separated into $2 P+1$ matrices containing the bits of the $i^{\text {th }}$ time interval:

$$
\mathbf{B}^{(i)}=\left(\begin{array}{ccc}
b_{1}^{(i)} \mathbf{I} & \cdots & 0 \\
\vdots & \ddots & \vdots \\
0 & \cdots & b_{K}^{(i)} \mathbf{I}
\end{array}\right),
$$

where $\mathbf{I}$ is an $L \times L$ identity matrix. The repetitive character of the above matrix with regard to the bits of one user is due to the multipath channel where for each user the same information passes through $L$ different paths.

The probability density function of the observed waveform (3) conditioned on $\mathcal{B}$ and $\boldsymbol{\alpha}$ yields the log-likelihood function which can be written (up to an additive constant) as [24]

$$
\Lambda(\mathcal{B}, \boldsymbol{\alpha})=\frac{2}{\mathcal{N}_{0}} \int \Re\left\{r(t) s^{*}(t, \mathcal{B})\right\} d t-\frac{1}{\mathcal{N}_{0}} \int|s(t, \mathcal{B})|^{2} d t,
$$

where $(\cdot)^{*}$ denotes the conjugate operation. Considering the particular modulation scheme used in the proposed system, the log-likelihood function (7) is equal to

$$
\begin{aligned}
\Lambda(\mathcal{B}, \boldsymbol{\alpha}) & =\sum_{i=-P}^{P} \frac{1}{\mathcal{N}_{0}}\left(2 \Re\left\{\boldsymbol{\alpha}^{\mathrm{H}} \mathbf{B}^{(i)} \mathbf{z}^{(i)}\right\}\right. \\
& \left.-\sum_{i^{\prime}=-P}^{P} \boldsymbol{\alpha}^{\top} \mathbf{B}^{(i)} \mathbf{R}^{\left(i-i^{\prime}\right)} \mathbf{B}^{\left(i^{\prime}\right)} \boldsymbol{\alpha}^{*}\right),
\end{aligned}
$$

where $(\cdot)^{\mathrm{H}}$ denotes the conjugate transpose and $\mathbf{z}^{(i)}=$ $\left(z_{1,1}^{(i)}, z_{1,2}^{(i)}, \ldots, z_{1, L}^{(i)}, z_{2,1}^{(i)}, \ldots, z_{K, L}^{(i)}\right)^{\top}$ is the vector of outputs of the $K L$ matched filters at the end of the $i^{t h}$ bit interval. Thus, the element

$$
z_{k, l}^{(i)}=\int_{-\infty}^{+\infty} r(t) a_{k}\left(t-\tau_{k, l}-i T_{b}\right) d t
$$

is the output of the filter matched to the $k^{\text {th }}$ user's signaling waveform passing through the $l^{\text {th }}$ path. In the likelihood function (8), the $K L \times K L$ matrix

$$
\begin{aligned}
\mathbf{R}^{(i)}= & \left(\begin{array}{ccc}
\mathbf{R}_{1,1}^{(i)} & \cdots & \mathbf{R}_{1, K}^{(i)} \\
\vdots & \ddots & \vdots \\
\mathbf{R}_{K, 1}^{(i)} & \cdots & \mathbf{R}_{K, K}^{(i)}
\end{array}\right) \text { with } \\
\mathbf{R}_{k, k^{\prime}}^{(i)} & =\left(\begin{array}{ccc}
R_{k, k^{\prime}}(1,1, i) & \cdots & R_{k, k^{\prime}}(1, L, i) \\
\vdots & \ddots & \vdots \\
R_{k, k^{\prime}}(L, 1, i) & \cdots & R_{k, k^{\prime}}(L, L, i)
\end{array}\right)
\end{aligned}
$$

represents the cross-correlation functions between the spreading signals, that is, the element

$$
R_{k, k^{\prime}}\left(l, l^{\prime}, i\right)=\int_{-\infty}^{+\infty} a_{k}\left(t-\tau_{k, l}\right) a_{k^{\prime}}\left(t+i T_{b}-\tau_{k^{\prime}, l^{\prime}}\right) d t
$$

defines the correlation between the $k^{t h}$ and the $\left(k^{\prime}\right)^{t h}$ user with respect to the $l^{t h}$ and the $\left(l^{\prime}\right)^{t h}$ path. It should be noted that the modulation signals are time-limited to $\left[0, T_{b}\right]$ and the propagation delays are ordered. Therefore, the cross-correlation functions $R_{k, k^{\prime}}\left(\cdot, \cdot, i-i^{\prime}\right)$ are zero when $\left|i-i^{\prime}\right|>2$. This fact will simplify the evaluation of the likelihood function since the summation over the bit index $i^{\prime}$ can be limited to the range $[i-2, i+2]$. From the likelihood function (8) it is clear that the outputs of a matrix of $K$ by $L$ matched filters or, equivalently, of a bank of $K$ Rake correlators with $L$ arms form the sufficient statistics for the demodulation of $\mathcal{B}$ as well as for the estimation of $\boldsymbol{\alpha}$

The direct maximum likelihood choices of $\boldsymbol{\alpha}$ and $\mathcal{B}$ can be described as

$$
(\hat{\mathcal{B}}, \hat{\boldsymbol{\alpha}})=\arg \left\{\max _{\mathcal{B}, \boldsymbol{\alpha}}\{\Lambda(\mathcal{B}, \boldsymbol{\alpha})\}\right\},
$$

where $\mathcal{B} \in\{-1,+1\}^{K \times(2 P+1)}$ and $\alpha \in \mathbb{C}^{K \times L}$. As noted in [21], the maximization of (12) is a highly nonlinear and complex problem that does not appear to be configurable as a dynamic program. The maximization of the generalized likelihood function assuming a statistical model for the channel coefficients can be viewed as

$$
\hat{\mathcal{B}}=\arg \left\{\max _{\mathcal{B} \in\{-1,+1\}^{K \times(2 P+1)}} E_{\boldsymbol{\alpha}}[\exp \{\Lambda(\mathcal{B}, \boldsymbol{\alpha})\}]\right\},
$$

where $E_{\boldsymbol{\alpha}}$ denotes the expectation with respect to the attenuation variables $\boldsymbol{\alpha}$. Moreover, it is difficult to obtain a solution to the above problem since the observation process $r(t)$ is dependent on a large set of unknown variables $\boldsymbol{\alpha}$. An alternative to (12) and (13) is to maximize the likelihood function using an iterative approach.

\section{Iterative Maximum Likelihood Algorithm}

In this section, we consider an iterative scheme for solving the maximum likelihood demodulation problem. In particular, we apply Gauss-Seidel iterations to solve the joint detection and estimation problem. Assuming that initial estimates for the channel parameters and data packets are available and are denoted by $\hat{\boldsymbol{\alpha}}(0)$ and $\hat{\mathcal{B}}(0)$, respectively, the iterations to obtain the $\left(m_{G S}+1\right)^{s t}$ estimates can be described as

$$
\begin{aligned}
& \hat{\boldsymbol{\alpha}}\left(m_{G S}+1\right)=\arg \left\{\max _{\boldsymbol{\alpha}}\left[\Lambda\left(\hat{\mathcal{B}}\left(m_{G S}\right), \boldsymbol{\alpha}\right)\right]\right\}, \text { and } \\
& \hat{\mathcal{B}}\left(m_{G S}+1\right)=\arg \left\{\max _{\mathcal{B}}\left[\Lambda\left(\mathcal{B}, \hat{\boldsymbol{\alpha}}\left(m_{G S}\right)\right)\right]\right\},
\end{aligned}
$$

where $\hat{\boldsymbol{\alpha}}\left(m_{G S}\right)$ and $\hat{\mathcal{B}}\left(m_{G S}\right)$ are the $\left(m_{G S}\right)^{t h}$ estimates of the unknown parameters. Figure 2 depicts the multiuser and multipath receiver with the proposed iterative processing scheme. Note that there is always a sign ambiguity when one considers joint data demodulation and phase estimation in BPSK systems. This sign ambiguity can easily be resolved by short predefined bit-patterns (e.g., a preamble having a length of one or more bits). We should emphasize that the short preamble is used to resolve the sign ambiguity and not to yield precise estimates for the channel parameters. More information on this implementation 


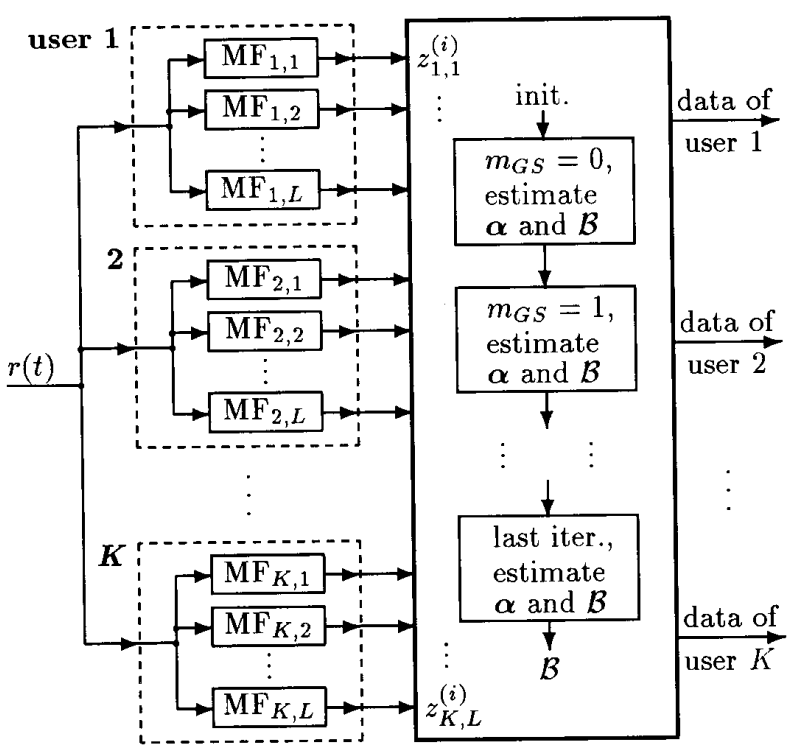

Fig. 2. Block diagram of the multiuser diversity receiver with batch processing. A bank of $K \times L$ matched filters (MF) provides the sufficient statistics.

related issue is given in Section VI. In the next section we concentrate on the first step of the Gauss-Seidel iteration.

\section{A. Estimation of Channel Parameters}

\section{A.1 Least Squares Solution}

The log-likelihood function (8) is quadratic in the channel parameters $\alpha_{k, l}$ 's. If estimates of the data packets are available, then the maximum likelihood estimation of $\alpha_{k, l}$ 's yields a least squares problem, that is, ${ }^{3}$

$$
\sum_{i=-P}^{P} \hat{\mathbf{B}}^{(i)} \mathbf{z}^{(i)}=\left(\sum_{i=-P}^{P} \sum_{i^{\prime}=i-2}^{i+2} \hat{\mathbf{B}}^{(i)} \mathbf{R}^{\left(i-i^{\prime}\right)} \hat{\mathbf{B}}^{\left(i^{\prime}\right)}\right) \boldsymbol{\alpha} .
$$

Therefore, solving the least squares problem in (15) to obtain an estimate of $\boldsymbol{\alpha}$ requires inverting the $K L \times K L$ matrix that multiplies $\boldsymbol{\alpha}$ in (15), provided that an inverse exists. The necessary matrix inversion may be impractical even for moderate data rates if the product of $K$ and $L$ is large.

\section{A.2 Expectation Maximization Algorithm}

The Expectation Maximization (EM) scheme is an iterative algorithm that may prove extremely useful for the estimation step of the problem in (14). The EM-algorithm was first proposed by Baum, et al. ${ }^{4}$ as an iterative method to find maximum likelihood estimates and was later expanded by Dempster, et al. ${ }^{4}$ Although this powerful technique was introduced in the statistics literature some twenty years

\footnotetext{
${ }^{3}$ The range of $i^{\prime}$ of the inner summation in (15) has to be kept within $[-P, P]$.
}

ago, it has only recently been applied to engineering problems [21, 25-28].

In this application of the EM-algorithm, the set of variables of interest is $\boldsymbol{\alpha}$ and the observed process is $r(t)$, which is also referred to as the incomplete data. The idea for the following decomposition is taken from [25] and is applied to the system model under consideration. The complete data is defined to be the decomposed signal $r(t)$, that is,

$$
\begin{aligned}
& \mathbf{X}(t)=\left(X_{1,1}(t), \ldots, X_{K, L}(t)\right)^{\top} \text { with } \\
& X_{k, l}(t)=\sum_{i=-P}^{P} \alpha_{k, l} \hat{b}_{k}^{(i)} a_{k}\left(t-\tau_{k, l}-i T_{b}\right)+N_{k, l}(t), \\
& \text { for } \forall k \text { and } l,
\end{aligned}
$$

where the random process modeling the observed noise $n(t)$ in (3) is denoted by $N(t)=\sum_{k=1}^{K} \sum_{l=1}^{L} N_{k, l}(t)$ and where all the decomposed noise processes $N_{k, l}(t)$ are chosen such that they are statistically independent. We first need to write the conditional log-likelihood function of the complete data as

$$
U\left(\boldsymbol{\alpha}, \boldsymbol{\alpha}^{\prime}\right)=E\left[\log \mathcal{P}_{\mathbf{X}}(\mathbf{X}, \boldsymbol{\alpha}) \mid r(t), \boldsymbol{\alpha}^{\prime}\right] .
$$

The expectation maximization algorithm is based on the following result from statistics which states that, considering $\hat{\boldsymbol{\alpha}}(m)$ and $\hat{\boldsymbol{\alpha}}(m+1)$ as two estimates of $\boldsymbol{\alpha}$,

$$
\begin{array}{ll}
\text { if } & \hat{\boldsymbol{\alpha}}(m+1)=\arg \left\{\max _{\boldsymbol{\alpha}}[U(\boldsymbol{\alpha}, \hat{\boldsymbol{\alpha}}(m))]\right\}, \\
\text { then } & \Lambda(\hat{\mathcal{B}}, \hat{\boldsymbol{\alpha}}(m+1)) \geq \Lambda(\hat{\mathcal{B}}, \hat{\boldsymbol{\alpha}}(m)) .
\end{array}
$$

Thus, we can solve the maximum likelihood problem with the above iterative procedure. Starting with an initial estimate for the parameters denoted by $\hat{\boldsymbol{\alpha}}(0)$, then at an instance of the algorithm, the E-step evaluates $U(\boldsymbol{\alpha}, \hat{\boldsymbol{\alpha}}(m))$ and the M-step maximizes $U(\boldsymbol{\alpha}, \hat{\boldsymbol{\alpha}}(m))$ with respect to $\boldsymbol{\alpha}$.

Since the complete data is a vector of independent Gaussian random processes conditioned on $r(t)$ and $\alpha$, we can write

$$
\begin{aligned}
& U(\boldsymbol{\alpha}, \hat{\boldsymbol{\alpha}}(m))=c-\sum_{k=1}^{K} \sum_{l=1}^{L} c_{k, l} \\
& \cdot \int\left|\hat{X}_{k, l}(t)-\sum_{i=-P}^{P} \alpha_{k, l} \hat{b}_{k}^{(i)} a_{k}\left(t-\tau_{k, l}-i T_{b}\right)\right|^{2} d t
\end{aligned}
$$

where $c$ and $c_{k, l}>0$ (for all $k$ and $l$ ) are real-valued constants and $\hat{X}_{k, l}(t)$ is the conditional expectation of $X_{k, l}(t)$ given $r(t)$ and $\hat{\boldsymbol{\alpha}}(m)$. At the $m^{\text {th }}$ step of the iteration, each $\hat{X}_{k, l}(t)$ is linear in $r(t)$ and can be written as

$$
\begin{gathered}
\hat{X}_{k, l}(t)=\sum_{i=-P}^{P} \hat{\alpha}_{k, l}(m) \hat{b}_{k}^{(i)} a_{k}\left(t-\tau_{k, l}-i T_{b}\right)+\beta_{k, l} \\
\cdot\left[r(t)-\sum_{i=-P}^{P} \sum_{k^{\prime}=1}^{K} \sum_{l^{\prime}=1}^{L} \hat{\alpha}_{k^{\prime}, l^{\prime}}(m) \hat{b}_{k^{\prime}}^{(i)} a_{k^{\prime}}\left(t-\tau_{k^{\prime}, l^{\prime}}-i T_{b}\right)\right]
\end{gathered}
$$

${ }^{4}$ References are provided in [21]. 
for all $k$ and $l$ where the positive coefficients $\beta_{k, l}$ are, in principle, free parameters that can be adjusted for improved performance subject to the condition that $\sum_{k=1}^{K} \sum_{l=1}^{L} \beta_{k, l}=1$. The power of the algorithm lies in the fact that the maximization of the $\log$-likelihood function over $\alpha_{k, l}$ 's for $k=1, \ldots, K$ and $l=1, \ldots, L$ has been decomposed into $K L$ independent and iterative procedures. Furthermore, from one iteration to the next there will be no decrease in the value of the log-likelihood function.

The maximization of the generalized log-likelihood function of the complete data (19) over the $\alpha_{k, l}$ 's is a simple linear least-squares problem once estimates of $\tau_{k, l}$ 's and $b_{k}^{(i)}$ 's are available. Therefore, taking the derivative of $U(\boldsymbol{\alpha}, \hat{\boldsymbol{\alpha}}(m))$ with respect to $\alpha_{k, l}$ and setting it to zero gives an estimate of $\alpha_{k, l}$ as

$$
\begin{aligned}
& \hat{\alpha}_{k, l}(m+1)=\frac{1}{(2 P+1) T_{b}} \\
& \cdot \sum_{i=-P}^{P} \hat{b}_{k, l}^{(i)} \int \hat{X}_{k, l}(t) a_{k}\left(t-\tau_{k, l}-i T_{b}\right) d t
\end{aligned}
$$

Now substituting (20) into (21) yields the following recursive estimator as:

$$
\begin{aligned}
& \hat{\boldsymbol{\alpha}}(m+1)=\hat{\boldsymbol{\alpha}}(m)+\frac{1}{(2 P+1) T_{b}} \boldsymbol{\beta} \\
& \quad\left\{\sum_{i=-P}^{P}\left(\hat{\mathbf{B}}^{(i)} \mathbf{z}^{(i)}-\sum_{i^{\prime}=i-2}^{i+2} \hat{\mathbf{B}}^{(i)} \mathbf{R}^{\left(i-i^{\prime}\right)} \hat{\mathbf{B}}^{\left(i^{\prime}\right)} \hat{\boldsymbol{\alpha}}(m)\right)\right\},
\end{aligned}
$$

where the diagonal matrix $\beta$ is defined as $\operatorname{diag}\left(\beta_{1,1}\right.$, $\left.\beta_{1,2}, \ldots, \beta_{1, L}, \beta_{2,1}, \ldots, \beta_{K, L}\right)$.

The EM-algorithm applied to the parameter estimation problem for the multiuser multipath system results in a set of recursive equations to solve for the attenuation variables. The algorithm can only guarantee convergence to a stationary point of the likelihood function under certain regularity conditions. The convergence properties also depend on the chosen initialization scheme. It is beyond the scope of this paper to investigate this subject in detail. We, however, present some numerical examples in Section VI.

\section{B. Detection Algorithm}

The second step in the Gauss-Seidel iteration (14) yields new bit estimates. In order to avoid the heavy computational requirement imposed by the optimum demodulation scheme, we implement a multistage detection algorithm. This suboptimum technique was first developed by Varanasi and Aazhang in [7] for additive white Gaussian channels. In this algorithm, the $\left(m^{\prime}+1\right)^{s t}$ stage estimate of $b_{k}^{(i)}$ for each $k=1, \ldots, K$ and $i=-P, \ldots, P$ is given by

$$
\hat{b}_{k}^{(i)}\left(m^{\prime}+1\right)=\arg \left\{\max _{\substack { b_{(i)}^{(i)} \in\{-1,+1\} \\
b_{k^{\prime}}^{\left(i^{\prime}\right)} \\
\begin{subarray}{c}{\prime^{\prime} \\
\hat{b}^{\prime}{ b _ { ( i ) } ^ { ( i ) } \in \{ - 1 , + 1 \} \\
b _ { k ^ { \prime } } ^ { ( i ^ { \prime } ) } \\
\begin{subarray} { c } { \prime ^ { \prime } \\
\hat { b } ^ { \prime } } }\end{subarray}}[\Lambda(\mathcal{B}, \hat{\boldsymbol{\alpha}})]\right\}
$$

where the maximization is performed by setting $b_{k^{\prime}}^{\left(i^{\prime}\right)}$ equal to its $\left(m^{\prime}\right)^{t h}$ stage estimate for all $k^{\prime} \neq k$ and $i^{\prime} \neq i$. Considering the form of the likelihood function, the multistage algorithm can also be expressed as

$$
\begin{aligned}
& \hat{b}_{k}^{(i)}\left(m^{\prime}+1\right)=\operatorname{sign}\left[\Re \left\{\hat{\boldsymbol{\alpha}}^{\mathrm{H}} \mathbf{D}_{k}\right.\right. \\
& \left.\left.\cdot\left(\mathbf{z}^{(i)}-\sum_{i^{\prime}=i-2}^{i+2} \mathbf{R}^{\left(i-i^{\prime}\right)} \mathbf{I}_{k}^{\left(i-i^{\prime}\right)} \hat{\mathbf{B}}^{\left(i^{\prime}\right)}\left(m^{\prime}\right) \hat{\boldsymbol{\alpha}}\right)\right\}\right]
\end{aligned}
$$

where $\mathbf{D}_{k}=\operatorname{diag}\left\{d_{1,1}, d_{1,2}, \ldots, d_{1, L}, d_{2,1}, \ldots, d_{K, L}\right\}$ is a $K L \times K L$ diagonal matrix with all elements equal to zero except for $d_{k, 1}=d_{k, 2}=\ldots=d_{k, L}=1$. In (24) the $K L \times K L$ diagonal matrix $\mathbf{I}_{k}^{(i)}$ is defined as $\mathbf{I}$ for all $i \neq 0$ and $\mathbf{I}_{k}^{(0)}=\mathbf{I}-\mathbf{D}_{k}$ for all $k$ with $\mathbf{I}$ denoting the identity matrix. Analogous to (6), the matrix $\hat{\mathbf{B}}^{(i)}\left(m^{\prime}\right)$ is defined for the estimated bits $\hat{b}_{k}^{(i)}\left(m^{\prime}\right)$ of the previous stage. In (24), the diversity is processed according to the maximal ratio combining scheme [24] since the matched filter samples $\mathbf{z}^{(i)}$ are weighted according to the path attenuation. The summation over $i^{\prime}$ in (24) represents the estimated interference of the $k^{\text {th }}$ user at the $i^{\text {th }}$ bit interval and is subtracted from the test statistics prior to the hard decision in the $\left(m^{\prime}+1\right)^{s t}$ stage.

For simplicity, the decision from a conventional singleuser Rake receiver is used as an initial estimate for the iterative scheme. Therefore, the first stage estimate of the multistage algorithm is

$$
\hat{b}_{k}^{(i)}(0)=\operatorname{sign}\left[\Re\left\{\hat{\boldsymbol{\alpha}}^{\mathrm{H}} \mathbf{D}_{k} \mathbf{z}^{(i)}\right\}\right] .
$$

\section{Sequential Implementation}

The advantages of sequential algorithms over batch algorithms, which process the received data as a whole, are higher computational efficiency and less allocated memory. The maximum likelihood solution proposed for estimation of the channel parameters is a batch algorithm. It is fortunate that the presented multiuser data detection scheme lends itself to a sequential configuration. In this section, we derive a sequential algorithm for estimating $\boldsymbol{\alpha}$ which may prove more practical especially for applications where the channel coefficients change gradually during transmission.

The need for formulating a sequential EM-algorithm has been addressed in a number of applications [29,30]. In most cases, it is only possible to derive a sequential algorithm that approximates the EM-algorithm [30]. The basic idea is to update the estimates of the attenuation variables as new observation data $\mathbf{z}^{(i)}$ becomes available and to convert the iteration index $m$ to the time related index $i$. Before formulating the sequential EM-algorithm, we first look at the following example where the $(i+1)^{s t}$ estimate is calculated as $\hat{\theta}_{i+1}=\hat{\theta}_{i}+\sum_{n=0}^{i} y_{n} /(i+1)$ for all positive integers $i$. The new parameter estimate is based on the previous one and the past $(i+1)$ observed data $y_{0}, y_{1}, \ldots, y_{i}$. It is easy 
to see that $\hat{\theta}_{i+1}$ can be calculated sequentially as

$$
\hat{\theta}_{i+1}=\hat{\theta}_{i}+\frac{i}{i+1}\left(\hat{\theta}_{i}-\hat{\theta}_{i-1}\right)+\frac{1}{i+1} y_{i}
$$

Applying the above technique to the problem in (22) yields the following sequential algorithm for updating the estimates of the attenuation parameters ${ }^{5}$

$$
\begin{aligned}
\hat{\boldsymbol{\alpha}}(i+1)=\hat{\boldsymbol{\alpha}}(i)+\frac{i+P}{i+P+1}(\hat{\boldsymbol{\alpha}}(i)-\hat{\boldsymbol{\alpha}}(i-1)) \\
+\frac{1}{(i+P+1) T_{b}} \boldsymbol{\beta} \\
\quad\left(\hat{\mathbf{B}}^{(i)} \mathbf{z}^{(i)}-\sum_{i^{\prime}=i-2}^{i+2} \hat{\mathbf{B}}^{(i)} \mathbf{R}^{\left(i-i^{\prime}\right)} \hat{\mathbf{B}}^{\left(i^{\prime}\right)} \hat{\boldsymbol{\alpha}}(i)\right),
\end{aligned}
$$

for $i=-P, \ldots, P-1$. The initial estimates $\hat{\boldsymbol{\alpha}}(-P)$ can either be obtained through a preamble or set to a predefined value (e.g., to zero or to the estimated value obtained from the previously demodulated data packet). The recursive scheme in (27) can be simplified further by approximating the time varying filter coefficients with constants $\gamma_{1}$ and $\gamma_{2}$ as

$$
\begin{aligned}
\hat{\boldsymbol{\alpha}}(i+1) & =\hat{\boldsymbol{\alpha}}(i)+\gamma_{2}(\hat{\boldsymbol{\alpha}}(i)-\hat{\boldsymbol{\alpha}}(i-1)) \\
& +\gamma_{1}\left(\hat{\mathbf{B}}^{(i)} \mathbf{z}^{(i)}-\sum_{i^{\prime}=i-2}^{i+2} \hat{\mathbf{B}}^{(i)} \mathbf{R}^{\left(i-i^{\prime}\right)} \hat{\mathbf{B}}^{\left(i^{\prime}\right)} \hat{\boldsymbol{\alpha}}(i)\right) .
\end{aligned}
$$

Such a sequential updating process allows the receiver to adapt to the time-variant behavior of the channel unless the coherence time is too small for the particular data rate. When $\gamma_{2}$ is set to zero, the estimator becomes a first order filter. It is interesting to note that in this case the sequential estimator resembles recursive algorithms which are often used in solving least squares problems based on stochastic gradients [31].

\section{Performance Analysis}

The structure of the proposed receiver is based on a joint estimation and detection algorithm. The variance of the estimator of $\boldsymbol{\alpha}$ can be considered as a good performance criterion for the estimation algorithm whereas the average bit-error probability quantifies the performance of the detector. This section contains analytical results on the variance of the estimated channel coefficients and on the biterror probability, as well as numerical results from Monte Carlo simulation of the proposed receiver.

\section{A. Lower Bound on the Variance of the Parameter Esti- mator}

The Cramér-Rao Inequality [24] provides a lower bound for the variance of the unbiased estimate of an unknown parameter. If this variance equals the bound, the corresponding estimator is called efficient. At this point, it is

${ }^{5}$ For real implementations some additional short delays have to be introduced to resolve the non-causality, i. $\epsilon$, to avoid bits being indexed by $i^{\prime}$ that are subject to be demodulated in a time instant ahead. appropriate to concentrate on the estimation of $\boldsymbol{\alpha}$. Therefore, we assume that the data packets $\mathcal{B}$ are known a priori, or have been correctly detected. The unbiasedness of the least squares estimates $\hat{\alpha}_{k, l}$ can be verified by inserting the vector $\mathbf{z}^{(i)}$ with the elements as defined by (9) into (15). When calculating the variance of the estimation error, it is convenient to split the attenuation variables into their real and imaginary parts, i.e., $\alpha_{k, l}=\alpha_{k, l}^{(c)}+j \alpha_{k, l}^{(s)}$. The Cramér-Rao lower bound can be computed for both parts with

$$
E\left[\left|\hat{\alpha}_{k, l}-\alpha_{k, l}\right|^{2}\right] \geq \frac{-1}{E\left[\frac{\partial^{2} \Lambda(\mathcal{B}, \boldsymbol{\alpha})}{\partial \alpha_{k, l}^{(c) 2}}\right]}+\frac{-1}{E\left[\frac{\partial^{2} \Lambda(\mathcal{B}, \boldsymbol{\alpha})}{\partial \alpha_{k, l}^{(s) 2}}\right]}
$$

By computing the second derivatives of the log-likelihood function (8) and expressing the attenuation variables by the channel coefficients (5), we obtain the second moment for the error of the estimated channel coefficient

$$
E\left[\left|\hat{h}_{k, l}-h_{k, l}\right|^{2}\right] \geq \frac{\mathcal{N}_{0}}{E_{b}} \cdot \frac{1}{2 P+1}, \forall k, \forall l,
$$

which is also termed as the mean square error (MSE) of $h_{k, l}$. Clearly, our performance criterion decreases with increasing $E_{b} / \mathcal{N}_{0}$ or packet length $2 P+1$. For an expanding observation time, this behavior is encountered in many estimation problems provided that the channel is timeinvariant.

\section{B. Lower Bound for the Average Bit-Error Probability}

A lower bound for the average bit-error probability can be obtained by simply considering the average error probability of a single-user (i.e., $K=1$ ) multipath system with perfect estimates of the channel parameters. In this case, it is easy to show that the average bit-error probability of the desired user (that is user 1 in our examples) in a multiuser system is lower bounded by: ${ }^{6}$

$$
\begin{aligned}
& \bar{P}_{e}(1) \geq \frac{1}{2} \\
& \cdot E\left\{\operatorname{erfc}\left(\sqrt{\frac{E_{b}}{\mathcal{N}_{0}}} \sqrt{\sum_{l=1}^{L} \sum_{l^{\prime}=1}^{L} \Re\left\{h_{1, l}^{*} h_{1, l^{\prime}}\right\} \frac{R_{1,1}\left(l, l^{\prime}, 0\right)}{T_{b}}}\right)\right\} .
\end{aligned}
$$

A looser lower bound for the average bit-error probability than (31) follows by considering only the dominant terms in the double summation in (31) as

$$
\bar{P}_{e}(1) \geq \frac{1}{2} \operatorname{erfc}\left(\sqrt{\frac{E_{1}}{\mathcal{N}_{0}}}\right) \text { with } E_{k}=\sum_{l=1}^{L} E_{b}\left|h_{k, l}\right|^{2}, \forall k .
$$

The expression of the received bit energy of the $k^{\text {th }}$ user in (32) is useful to describe the near-far effect, which is formulated by choosing different path attenuation variables. These results emphasize the importance of exploiting the diversity by processing all the $L$ multipath signals

$$
{ }^{6} \operatorname{erfc}(x)=\frac{2}{\sqrt{\pi}} \int_{x}^{\infty} \exp \left(-t^{2}\right) d t
$$


to achieve performance close to standard BPSK systems. The simulation results presented in the following section illustrate that the proposed multiuser multipath receiver performs with an average bit-error probability close to the lower bound in (31).

\section{Numerical Results}

In the following numerical examples we examine the performance of the proposed receivers in a multiuser multipath environment. Extensive Monte Carlo simulations are carried out to approximate the average bit-error probability and the mean square error of the detection and estimation algorithms in various system conditions.

To better understand these results, it is necessary to describe the details of the underlying simulation model. In the examples considered, short predefined bit-patterns are periodically inserted in the transmitted data stream in order to resolve the phase ambiguity at the receiver. This side-information can also be used to obtain initial estimates of the unknown attenuation variables and data bits. For the batch scheme, we assume that the receiver knows the first bits $\mathbf{B}^{(-P)}$ of every packet. We propose the use of an uncomplicated scheme for obtaining the initial estimates of the attenuation parameters: $\hat{\boldsymbol{\alpha}}\left(m_{G S}=0\right)=\mathbf{B}^{(-P)} \mathbf{z}^{(-P)}$. The initial estimate of the data packet $\hat{\mathcal{B}}\left(m_{G S}=0\right)$ is obtained by applying a one-stage detector (25) based on the initial estimates of the attenuation parameters. ${ }^{7}$ It is natural to provide the estimation and detection algorithm of the Gauss-Seidel iteration (14) with the most current estimates of the variables of interest and, if the EM-scheme is applied to estimate the attenuation variables, to use the most current estimates to initialize the EM-iterations.

We further need to define all system parameters. For simplicity it is assumed that the absolute values of the channel coefficients are the same for each user. The received signal energies of all users remain constant through the transmission of a data packet. Although this approach does not incorporate any amplitude fluctuations (e.g., the effect of Rayleigh fading), it characterizes the momentary performance of the receiver for the channel constellation during the length of one packet. We have chosen spreading sequences with optimized correlation properties, that is, the set of $m$-sequences of length $N_{c}=31$ as tabulated in [32]. More precisely, the $k^{t h}$ sequence in the list is assigned to the $k^{t h}$ user. The phase components of the complex channel coefficients are generated to be independent and uniformly distributed on $[0,2 \pi)$. The delay values of the first path for all the users are modeled as independent and uniformly on the set $\left\{0, T_{c}, \ldots,\left(N_{c}-1\right) T_{c}\right\}$. The difference of the delays $\left(\tau_{k, 2}-\tau_{k, 1}\right), \forall k$, is generated similarly. However, to approximate a "typical" multipath channel $[16,17]$ we restrict to the values of the subset $\left\{T_{c}, \ldots, 4 T_{c}\right\}$.

\footnotetext{
${ }^{7}$ Some experiments have shown that the proposed iterative schemes converge well (with respect to the channel coefficients up to their sign) even when the estimated attenuation parameters are initially set to zero and the initial bit estimates are set to randomly generated binary values.
}

a)

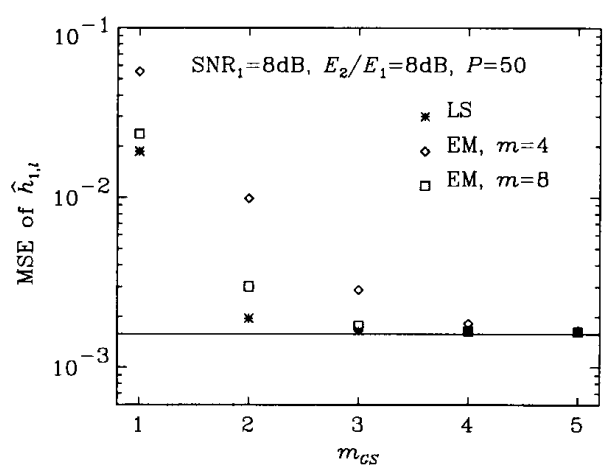

b)

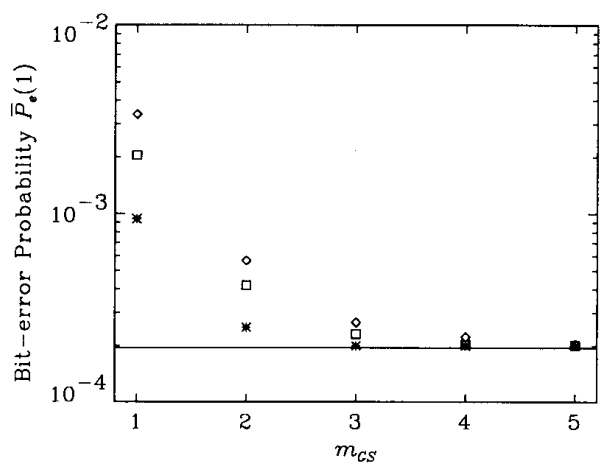

Fig. 3. Simulated MSE of the estimated channel coefficient $\hat{h}_{1, l}$ in a), and simulated bit-error probability $\bar{P}_{e}(1)$ in b) versus number of Gauss-Seidel iterations $m_{G S}$ for a two-user two-path system with $N_{c}=31$. The solid lines illustrate the lower-bounds.

\section{C.1 Performance of the Batch EM/Multistage Receiver}

In this section, we present simulation results on the performance of a receiver implementing the batch EMalgorithm and multistage detection algorithm for estimation of channel coefficients and data demodulation, respectively. In the EM-algorithm (22) all $\beta_{k, l}$ 's are designed to be equal. We consider a two-user system with two paths for the channel of each user, i.e., $K=2$ and $L=2$. The signal to noise ratio of the desired user $\left(\mathrm{SNR}_{1}=E_{1} / \mathcal{N}_{0}\right)$ is fixed at $8 \mathrm{~dB}$ and the ratio of the energy of user 2 to the desired user is $8 \mathrm{~dB}$. Figures $3 \mathrm{a}$ ) and $3 \mathrm{~b}$ ) depict the mean square error and the average bit-error probability, respectively, versus the number of Gauss-Seidel iterations $m_{G S}$ between the multistage detection and the estimation algorithms. The solid line in Fig. 3 a) illustrates the CramérRao lower bound (30) on the variance of the parameter estimator for the particular value of signal to noise ratio and packet length. In Fig. $3 \mathrm{~b}$ ), the solid line corresponds to the performance of a single-user multipath system (31) which is also a lower bound to $\bar{P}_{e}(1)$. These figures indicate that the EM-algorithm (22) with $m=8$ iterations achieves a mean square error and an average bit-error rate close to the least squares (LS) solution (15) which is computational more intensive. The performance of the proposed receiver in terms of the mean square error and $\bar{P}_{e}(1)$ approaches the corresponding lower bounds after $m_{G S}=4$ 
a)

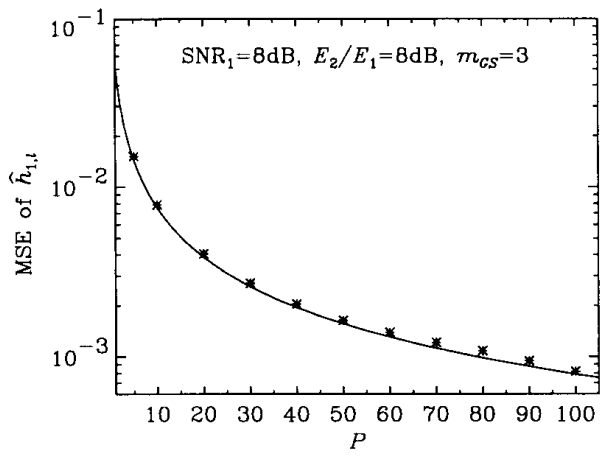

b)

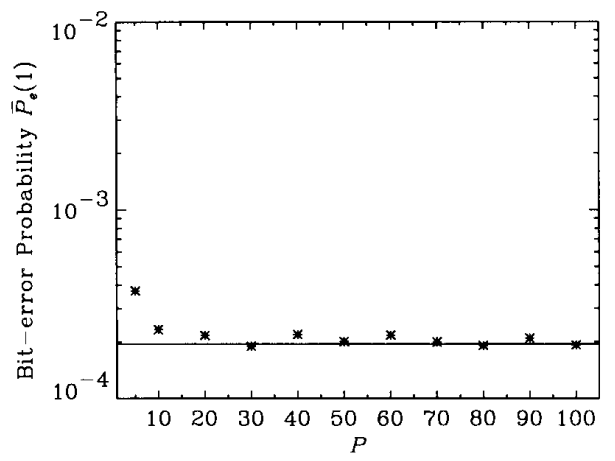

Fig. 4. Simulated MSE of the estimated channel coefficient $\hat{h}_{1, l}$ in a), and simulated bit-error probability $\bar{P}_{e}(1)$ in b) versus packet length $2 P+1$ for a two-user two-path system. The solid lines illustrate the lower-bounds.

Gauss-Seidel iterations. Therefore, Figs. 3 a) and 3 b) imply that the proposed estimator becomes "nearly" efficient and the multiuser detector rejects the multiple access interference after only 4 iterations between the estimation and detection processes.

Figure 4 captures the effects of the observation period $(2 P+1) T_{b}$ on the performance of a receiver with the least squares solution for parameter estimation and multistage algorithm for data demodulation. Figure 4 a) and 4 b) depict the mean square error and the average bit-error probability, respectively, versus the packet length for $m_{G S}=3$ iterations between the estimation and detection algorithms. Although the estimation error decreases with increasing $P$, the simulation results indicate that the bit-error probability reaches the lower bound for $P \geq 20$. Therefore, the trend in this figure justifies the choice of $P=50$ in the previous figure.

\section{C.2 Performance of the Sequential EM/Multistage Re- ceiver}

This section includes simulation results on the performance of a receiver implementing the sequential EMalgorithm for estimation of channel coefficients and the multistage detection algorithm for data demodulation. Once again we consider a two-user system with two paths for the channel of each user, i.e., $K=2$ and $L=2$. First

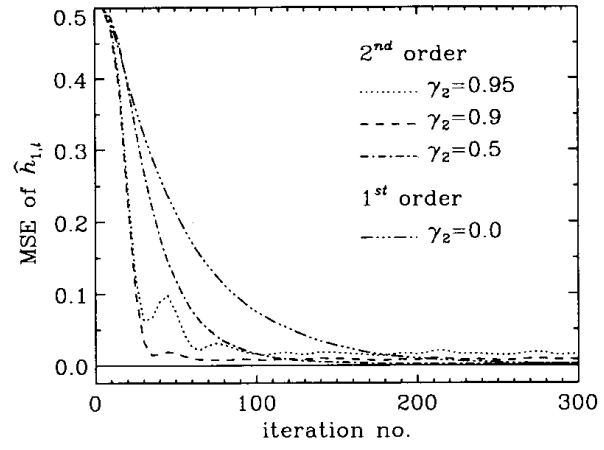

Fig. 5. Simulated MSE of $\hat{h}_{1, l}$ versus iteration number for the sequential implementation with $\gamma_{1}=0.01, E_{2} / E_{1}=8 \mathrm{~dB}$ and $\mathrm{SNR}_{1}=8 \mathrm{~dB}$. Two-user two-path system.

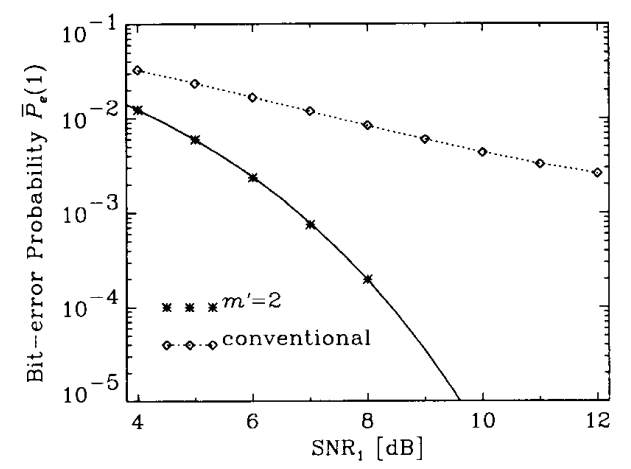

Fig. 6. Simulated probability of bit-error $\bar{P}_{e}(1)$ versus $\mathrm{SNR}_{1}$ for a fixed $E_{2} / E_{1}=8 \mathrm{~dB}$ of the sequential implementation compared to the calculated error probability (31) (solid line). Two-user two-path system.

we investigate the convergence properties of the sequential estimation scheme (28) and then evaluate the bit-error rate of the demodulator in the steady state. Figure 5 depicts the mean square error versus the number of iterations (i.e., the number of data bits) for various values of the filter parameter $\gamma_{2}$. The signal to noise ratio of the desired user $\left(\mathrm{SNR}_{1}\right)$ is fixed at $8 \mathrm{~dB}$ and the ratio of the energy of user 2 to the desired user is $8 \mathrm{~dB}$. Figure 5 includes the MSE values for both second order as well as first order filters (i.e., $\left.\gamma_{2}=0.0\right)$. As expected the first order filter yields a slightly lower steady state error with slower transient behavior than the second order filter. This example illustrates that, for most proper filter coefficient values, the sequential EMalgorithm achieves an acceptable mean square error after 100 data bits.

The steady state performance of the multiuser multipath receiver is presented in Fig. 6 where the average bit-error probability is plotted versus signal to noise ratio of user 1 with $E_{2} / E_{1}$ at $8 \mathrm{~dB}$. The performance of the proposed sequential receiver with $\gamma_{1}=0.01$ and $\gamma_{2}=0.0$ is compared to that of the conventional Rake receiver with no postprocessing. As expected the conventional Rake receiver is 


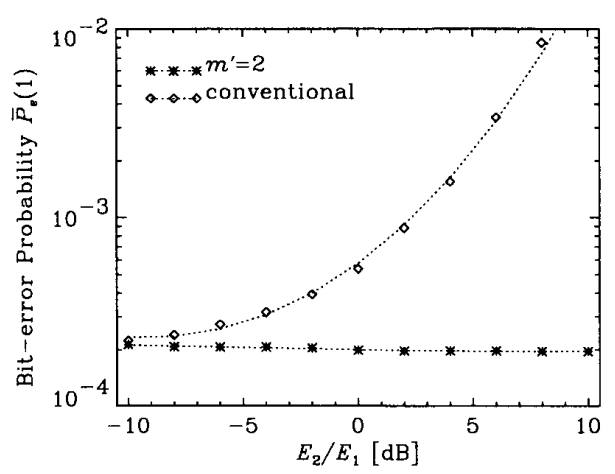

Fig. 7. Probability of bit-error $\bar{P}_{e}(1)$ versus $E_{2} / E_{1}$ for a fixed $\mathrm{SNR}_{1}=8 \mathrm{~dB}$. Two-user two-path system.

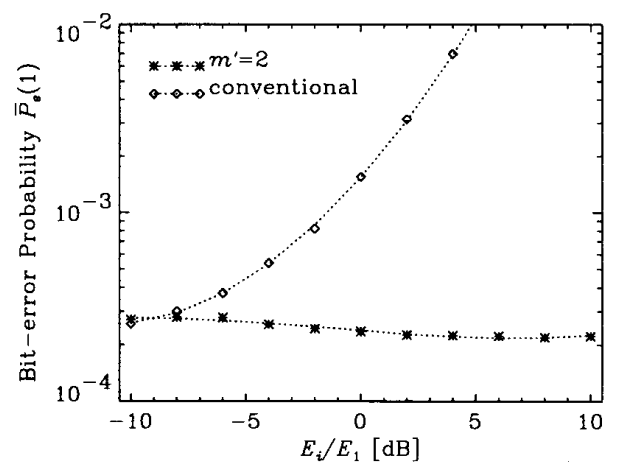

Fig. 8. Probability of bit-error $\bar{P}_{e}(1)$ versus $E_{2} / E_{1}=E_{3} / E_{1}$ for a fixed $\mathrm{SNR}_{1}=8 \mathrm{~dB}$. Three-user two-path system.

multiple access limited, that is, the performance does not improve with increase signal to noise ratio since the dominant interference is due to the second user. However, the multiuser receiver performs close to the lower bound and is successful in rejecting the interference from the second user while estimating the channel parameters. Figures 5 and 6 verify that the proposed estimator becomes efficient and the multiuser detector rejects the multiple access interference after only about 100 iterations between the estimation and detection processes which corresponds to 100 data bits.

Figures 7 and 8 depict the average bit-error probability versus the energy ratio of the received signal of the interfering users to that of the first user for a two-user and three-user system, respectively. The signal to noise ratio of user one is fixed at $8 \mathrm{~dB}$ in both figures and we have as before $\gamma_{1}=0.01$ and $\gamma_{2}=0.0$. Once again, the impact of strong interferers on the error rate is very small while there is a slight decrease in performance in the example with three users in Fig. 8 for small values of $E_{i} / E_{1}$ for $i=2$ and 3 . Fortunately, in this range of signal to noise ratios, the near-far problem does not arise and both the conventional and the multiuser receiver perform well.

\section{Conclusions}

This paper considers the problem of multiuser detection in multipath fading channels. A number of users are assumed to share a CDMA channel that exhibits frequency selective fading. The data demodulation problem for a multiuser system is complicated since, apart from additive white Gaussian noise, the users encounter multiple access interference and multipath propagation. The attenuation variables are assumed to be unknown. However, the spreading codes and the relative propagation delays of the various paths of all users are known to the receiver.

In order to manage the complexity of the direct maximum likelihood approach, an iterative procedure, known as the Gauss-Seidel algorithm, was proposed. The latter has been proven effective in many applications in optimization. In particular, the EM technique was used to estimate the attenuation parameters while the multistage scheme was implemented to demodulate the data. The resulting scheme for joint parameter estimation and data detection is a batch algorithm. To reduce the implementation cost, an algorithm was then derived which sequentially processes the incoming data.

The performance of these schemes, presented in Section VI, was analyzed via Monte Carlo computer simulations. The Cramér-Rao Inequality provided a lower bound on the error of the estimated channel coefficients. This bound is almost reached in a few Gauss-Seidel iterations in the batch $\mathrm{EM} /$ multistage scheme and in a reasonable time in the sequential EM/multistage scheme as indicated by the numerical results. The estimation errors of the attenuation variables are also shown to be reasonable to the extent that the corresponding degradation on the performance of the multiuser detector is negligible. In both batch and sequential examples, the probability of bit-error of the desired user is almost independent of the strength of the interfering signals. In addition, the diversity feature of the Rake receiver is preserved. It is clear from the analytical results and was confirmed by numerical examples that the detector is capable of adapting to the channel and eliminating the near-far effect.

The trade off between the batch scheme and the sequential scheme is complexity versus performance. The sequential receiver is slightly inferior in performance to the batch algorithm. However, the sequential scheme is much easier to implement and, because of its adaptive nature, it may be more appropriate for tracking varying channel parameters.

\section{ACKNOWLEDGMENTS}

The authors would like to acknowledge the encouragement and the continuous support of Professor Dr. Peter Leuthold, Director of the Communication Technology Laboratory at ETH-Zürich. They are also grateful to Dr. Jürgen Kemper for his help and support throughout the course of this work.

\section{REFERENCES}

[1] R. L. Pickholtz, L. B. Milstein, and D. L. Schilling, "Spread spectrum for mobile communications," IEEE Trans. Veh. Technol., 
vol. 40, pp. 313-322, May 1991.

[2] K. S. Gilhousen, I. M. Jacobs, R. Padovani, A. J. Viterbi, L. A. Weaver, Jr., and C. E. Wheatley III, "On the capacity of a cellular CDMA system," IEEE Trans. Veh. Technol., vol. VT40, pp. 303-312, May 1991.

[3] J. E. Mitzlaff, "Radio propagation and anti-multipath techniques in the WIN environment," IEEE Network Mag., vol. 5, pp. 2126, Nov. 1991.

[4] S. Verdú, "Minimum probability of error for asynchronous Gaussian multiple-access channels," IEEE Trans. Inform. Theory, vol. IT-32, pp. 85-96, Jan. 1986.

[5] H. V. Poor and S. Verdú, "Single-user detectors for multiuser channels," IEEE Trans. Commun., vol. COM-36, pp. 50-60, Jan. 1988.

[6] R. Lupas and S. Verdú, "Linear multiuser detectors for synchronous code-division multiple-access channels," IEEE Trans. Inform. Theory, vol. IT-35, pp. 123-136, Jan. 1989.

[7] M. K. Varanasi and B. Aazhang, "Multistage detection in asynchronous code-division multiple-access communications," IEEE Trans. Commun., vol. COM-38, pp. 509-519, Apr. 1990

[8] B. Aazhang, B.-P. Paris, and G. Orsak, "Neural networks for multiuser detection in code-division multiple-access communications," IEEE Trans. Commun., vol. COM-40, pp. 1212-1222, July 1992.

[9] Z. Xie, C. K. Rushforth, R. T. Short, and T. K. Moon, "Joint signal detection and parameter estimation in multiuser communications," IEEE Trans. Commun., vol. COM-41, pp. 1208-1216, Aug. 1993.

[10] U. Madhow and M. L. Honig, "MMSE interference suppression for direct-sequence spread-spectrum CDMA," (submitted to IEEE Trans. Commun.).

[11] Z. Zvonar and D. Brady, "Optimum detection in asynchronous multiple-access multipath Rayleigh fading channels," in Proc. of 26th Annual Conference on Information Sciences and Systems, (Princeton University), Mar. 1992.

[12] M. K. Varanasi and S. Vasudevan, "Multiuser detectors for synchronous CDMA communication over non-selective Rician fading channels," IEEE Trans. Commun., vol. COM-42, pp. 711722, Feb./Mar./Apr. 1994.

[13] A. Klein and P. W. Baier, "Linear unbiased data estimation in mobile radio systems applying CDMA," IEEE J. Select. Areas Commun., vol. SAC-11, pp. 1058-1066, Sept. 1993.

[14] Y. C. Yoon, R. Kohno, and H. Imai, "A spread-spectrum multiaccess system with cochannel interference cancellation for multipath fading channels," IEEE J. Select. Areas Commun., vol. SAC-11, pp. 1067-1075, Sept. 1993.

[15] S. S. H. Wijayasuriya, J. P. McGeehan, and G. H. Norton, "RAKE decorrelating receiver for DS-CDMA mobile radio networks," Electronics Letters, vol. 29, pp. 395-396, Feb. 18, 1993.

[16] T. S. Rappaport, S. Y. Seidel, and K. Takamizawa, "Statistical channel impulse response models for factory and open plan building radio communication system design," IEEE Trans. Commun., vol. COM-39, pp. 794-807, May 1991.

[17] A. Radović and E. Zollinger, "Measured time-variant characteristics of radio channels in the indoor environment," in Proc. of Mobile Radio Conf. (MRC), (Nice, France), pp. 267-274, Nov 13-15, 1991.

[18] G. L. Turin, "Introduction to spread-spectrum antimultipath techniques and their application to urban digital radio," Proc. IEEE, vol. 68, pp. 328-353, Mar. 1980.

[19] U. Fawer, "A coherent spread-spectrum diversity-receiver with AFC for multipath fading channels," IEEE Trans. Commun. vol. COM-42, pp. 1300-1311, Feb./Mar./Apr. 1994.

[20] J. M. Ortega and W. C. Rheinboldt, Iterative Solution of Nonlinear Equations in Several Variables. New York, NY: Academic Press, 1970.

[21] H.V. Poor, "On parameter estimation in DS/SSMA formats," in Lecture Notes in Control and Information Sciences - Advances in Communications and Signal Processing, pp. 59-70, SpringerVerlag, 1989.

[22] A. Radović and B. Aazhang, "Iterative algorithms for joint data detection and delay estimation for code division multiple access communication systems," in Proc. of the 31st Annual Allerton Conference on Communication, Control, and Computing, (Allerton House, Monticello, IL), Sept. 29-Oct. 1, 1993.

[23] S. E. Bensley and B. Aazhang, "Subspace-based delay estimation for CDMA communication systems," in Proc. of the Int. Symp. on Inf. Theory, (Trondheim, Norway), p. 138, June 27 - July 1, 1994.

[24] H. L. van Trees, Detection, Estimation, and Modulation Theory, Part I. New York, NY: John Wiley \& Sons, 1968

[25] M. Feder and E. Weinstein, "Parameter estimation of superimposed signals using the EM algorithm," IEEE Trans. Acoust. Speech, Signal Processing, vol. ASSP-36, pp. 477-489, Apr. 1988.

[26] M. Feder and J. A. Catipovic, "Algorithms for joint channel estimation and data recovery - application to equalization in underwater communications," IEEE J. Oceanic Eng., vol. OE16, pp. 42-55, Jan. 1991.

[27] M. S. Segal, E. Weinstein, and B. R. Musicus, "Estimatemaximize algorithms for multichannel time delay and signal estimation," IEEE Trans. Signal Processing, vol. SP-39, pp. 1-16, Jan. 1991.

[28] C. N. Georghiades and D. L. Snyder, "The expectationmaximization algorithm for symbol unsynchronized sequence detection," IEEE Trans. Commun., vol. COM-39, pp. 54-61, Jan. 1991.

[29] D. M. Titterington, "Recursive parameter estimation using incomplete data," J. Royal Statist. Soc., Ser. B, vol. 46, no. 2, pp. 257-267, 1984.

[30] M. Feder, E. Weinstein, and A. V. Oppenheim, "A new class of sequential and adaptive algorithms with application to noise cancellation," in Proc. of ICASSP'88, (New York, NY), pp. 557560, April 11-14, 1988.

[31] M. L. Honig and D. G. Messerschmitt, Adaptive Filters. Boston, MA: Kluwer, 1984

[32] M. B. Pursley and H. F. A. Roefs, "Numerical evaluation of correlation parameters for optimal phases of binary shift-register sequences," IEEE Trans. Commun., vol. COM-27, pp. 15971604, Oct. 1979.

Urs Fawer (S'86-M'94) was born in Laufenburg (AG), Switzerland in 1962. He received the Diploma in electrical engineering and the Ph.D. degree (Dr. sc. techn.) both from the Swiss Federal Institute of Technology (ETH), Zürich, in 1986 and 1993, respectively.

From 1987 to 1993, he was a Research Assistant in the Communication Technology Laboratory (Institut für Kommunikationstechnik) at ETH, Zürich, Switzerland. In September 1993, he joined Rice University, Houston, Texas, as a Postdoctoral Fellow. His one-yea stay was supported by the Swiss National Science Foundation. Since 1994, he is with Faselec AG (Philips Semiconductors), Zürich. His primary interests are in cellular mobile radio communications, integrated circuits for telecom applications, and specifically in digital synchronization algorithms, spread-spectrum communications, and multiuser detection.

Behnaam Aazhang (S'81-M'85-SM'91) was born in Bandar Anzali, Iran, on December 7, 1957. He received his B.S. (with highest honors), M.S., and Ph.D. degrees in Electrical and Computer Engineering from University of Illinois at Urbana-Champaign in 1981, 1983, and 1986 respectively.

From 1981 to 1985 , he was a Research Assistant in the Coordinated Science Laboratory, University of Illinois. In August 1985, he joined the faculty of Rice University, Houston, Texas, where he is now an Associate Professor of Electrical and Computer Engineering. He has been a Visiting Professor at IBM Federal Systems Company, Houston, Texas, the Institut für Kommunikationstechnik at Swiss Federal Institute of Technology (ETH), Zürich, Switzerland, the Telecommunications Laboratory at University of Oulu, Oulu, Finland, and at the U.S. Air Force Phillips Laboratory, Albuquerque, New Mexico. His research interests are in the areas of communication theory, information theory, and their applications with emphasis on radio and optical multiple access communications; cellular mobile radio communications; discrete event simulations and perturbation analysis; and data driven nonlinear modeling.

Dr. Aazhang is a recipient of the Alcoa Foundation Award 1993 the NSF Engineering Initiation A ward 1987-1989, and the IBM Graduate Fellowship 1984-1985, and is a member of Tau Beta Pi and Eta Kappa Nu. He is currently serving as the Editor for Spread Spectrum Networks of IEEE Transactions on Communications. He has served as the Secretary of the Information Theory Society 1990-1993 and as the Publications Chairman of the 1993 IEEE International Symposium on Information Theory, San Antonio, Texas. 